\title{
Phenethyl isothiocyanate enhances adriamycin-induced apoptosis in osteosarcoma cells
}

\author{
QIE FAN, XINLI ZHAN, ZENGMING XIAO and CHONG LIU \\ Department of Spinal Surgery, The First Affiliated Hospital of Guangxi Medical University, \\ Nanning, Guangxi Zhuang Autonomous Region 530021, P.R. China
}

Received September 23, 2014; Accepted June 11, 2015

DOI: $10.3892 / \mathrm{mmr} .2015 .4187$

\begin{abstract}
Adriamycin (ADM) is a first-line agent administered during the therapeutic regimes against osteosarcoma. Clinical administration of ADM produces systemic toxicity and resistance in patients, which restricts its applicability. In the present study the effects of phenethyl isothiocyanate (PEITC) on ADM-induced apoptosis in osteosarcoma cells was evaluated. Using U2-OS osteosarcoma cell line cells, treatment with PEITC or ADM for $24 \mathrm{~h}$ was observed to dose-dependently inhibit proliferation of U2-OS cells with half maximal inhibitory concentration $\left(\mathrm{IC}_{50}\right)$ values of $5.33 \mu \mathrm{M}$ and $10.32 \mu \mathrm{g} / \mathrm{ml}$, respectively. When U2-OS cells were treated with a combination of the two agents, the inhibition was apparently enhanced, as the $\mathrm{IC}_{50}$ values decreased to $2 \mu \mathrm{M}$ for PEITC and $1 \mu \mathrm{g} / \mathrm{ml}$ for ADM. Flow cytometry and terminal deoxynucleotidyl transferase dUTP nick end labeling revealed that treatment with PEITC or ADM alone reduced the viability of the U2-OS cells. Furthermore, the viability of the U2-OS cells was additionally reduced when treatment was with PEITC and ADM together. Supporting this finding, the activity and expression of caspase-3 were observed to be enhanced in the U2-OS cells following treatment with either PEITC or ADM, or a combination of the two. These results clearly indicate that PEITC enhances ADM-induced apoptosis in osteosarcoma cells.
\end{abstract}

\section{Introduction}

Osteosarcoma is a rare type of sarcoma, however, it is the most common histological form of bone cancer, which is associated with a poor prognosis due to early pulmonary metastasis $(1,2)$. Although osteosarcoma occurs in patients of all ages, osteosarcoma is more common in children and comprises $2.4 \%$ of

Correspondence to: Dr Xinli Zhan, Department of Spinal Surgery, The First Affiliated Hospital of Guangxi Medical University, 22 Shuangyong Road, Nanning, Guangxi Zhuang Autonomous Region 530021, P.R. China

E-mail: xinlizhan56@sogou.com

Key words: phenethyl isothiocyanate, adriamycin, apoptosis, osteosarcoma all malignancies in pediatric patients worldwide (3). The cause of osteosarcoma is unclear and risk factors, such as irradiation and genetic influences contribute to the development of osteosarcoma (4).

Adriamycin (ADM) belongs to a group of chemotherapeutic agents, termed anthracycline antibiotics that are able to slow or stop the growth of cancer cells (5). It has been clinically applied to treat patients exhibiting a variety of types of cancer, including osteosarcoma. Similar to other chemotherapeutic agents, although it has a killing effect on cancer cells, ADM eventually becomes ineffective due to the cancer cells developing ADM resistance (6). In addition, the toxic side-effects reduce the success of the treatment in patients. Therefore, a strategy is necessary to sensitize ADM in order to decrease the dose required for administration in clinical practice.

Phenethyl isothiocyanate (PEITC) is a compound of naturally occurring isothiocyanates found in a variety of cruciferous vegetables and is produced by enzymatic conversion of glucosinolate. PEITC has been evaluated as a potential antitumor agent in various types of cancer. For example, PEITC regulates epigenetic process and inhibits histone deacetylases in prostate cancer, leukemia and myeloma cells (7-9). Increasing numbers of studies have demonstrated that PEITC inhibits cell proliferation and induces apoptosis in various types of tumor cell (10-13). Recently, PEITC has been shown to sensitize tumor cells to chemotherapeutic agents and synergistically enhance chemotherapeutic agent-induced apoptosis of cancer cells (14-18). However, it remains unknown as to whether PEITC influences ADM-induced apoptosis in osteosarcoma.

In the present study, U2-OS cells were used to determine the synergistic effects of PEITC and ADM treatment on osteosarcoma cells. The half maximal inhibitory concentration $\left(\mathrm{IC}_{50}\right)$ values of PEITC and ADM, either alone or in combination, against U2-OS cells were measured. In addition, the effects of PEITC and ADM treatment, either alone or in combination, on proliferation and apoptosis of U2-OS cells were assessed. Finally, the potential signaling pathways involved in PEITC and ADM-induced apoptosis of U2-OS cells were investigated.

\section{Materials and methods}

Cells and reagents. U2-OS cells were purchased from the Cell Bank of Chinese Academy of Sciences (Shanghai, China). An MTT cell counting kit, Annexin V-fluorescein isothiocyanate 
(FITC) and propidium iodide (PI) were purchased from Vazyme Biotech (Nanjing, China). 2-Phenylethyl Isothiocyanate was purchased from TSI Instrument (Beijing) Co., Ltd. (Shanghai, China). ADM was purchased from Sigma-Aldrich (St. Louis, MO, USA). 4',6-diamidino-2-phenylindole dihydrochloride (DAPI) and a caspase-3 activity assay kit were purchased from Beyotime Institute of Biotechnology (Haimen, China). A bicinchoninic acid assay (BCA) protein assay kit was purchased from Geneseed Biotech Co., Ltd. (Guanzhou, China). Rabbit anti-caspase-3 (1:1,000; cat. no. BS1518), rabbit anti-Fas (1:500; cat. no. BS1745), and rabbit anti-FasL (1:500; cat. no. BS1122; Bioworld Technology, Inc.) were purchased from Bioworld Technology Inc. (St. Louis Park, MN, USA). Mouse anti- $\beta$-actin antibodies (1:10,000; cat. no. DKM9001) were purchased from Sungene Biotech Co., Ltd. (Tianjin, China). Horseradish peroxidase-conjugated goat anti-rabbit (cat. no. LK2001) and anti-mouse (cat. no. KM9001) IgG $(\mathrm{H}+\mathrm{L})-\mathrm{HRP}$ secondary antibodies $(1: 10,000)$ were purchased from Sungene Biotech Co., Ltd.

MTT assay. The proliferation of U2-OS cells was measured using an MTT cell counting kit according to the manufacturer's instruction. U2-OS cells (100 $\mu$ l of the suspension) were harvested at the log phase and seeded into a 96 -well plate at $6 \times 10^{3}$ cells/well and cultured overnight at $37^{\circ} \mathrm{C}$ in a $5 \% \mathrm{CO}_{2}$ atmosphere and saturated humidity. Then, $100 \mu 1$ PEITC and $100 \mu \mathrm{l}$ ADM alone or in combination (100 $\mu 1$ total volume; 1:1) were added to the wells. Triplicate wells were set for each treatment. After $24 \mathrm{~h}, 20 \mu \mathrm{l}$ MTT solution $(5 \mathrm{mg} / \mathrm{ml} 0.5 \%$ MTT) was added into each well and incubated at $37^{\circ} \mathrm{C}$ for 4 h. Following washing with phosphate-buffered saline (PBS), dimethyl sulfoxide and glycine buffering solution (Takara Bio, Inc., Otsu, Japan) were added to stop the reaction. The plates were maintained at room temperature in the dark for $2 \mathrm{~h}$ and the optical density (OD) value at $490 \mathrm{~nm}$ was measured using a microtiter plate reader $\left(\right.$ CytoFluor $^{\circledR}$ Series 4000 Fluorescence Multi-Well Plate Reader; Applied Biosystems Life Technologies, Foster City, CA, USA).

Flow cytometry. U2-OS cells were digested with $0.05 \%$ trypsin/0.5 mM EDTA ( $\mathrm{pH} 8.0$ ) for $3 \mathrm{~min}$, harvested and washed with PBS. Cells were resuspended in $95 \mu$ l Annexin V staining buffer [10 $\mu \mathrm{M}$ HEPES (pH 7.4), $140 \mathrm{mM} \mathrm{NaCl}$, $2.5 \mathrm{mM} \mathrm{CaCl}_{2}$ ] (GE Healthcare Life Sciences, Piscataway, NJ, USA) and incubated with $5 \mu \mathrm{l}$ Annexin V-FITC for $15 \mathrm{~min}$ at $4^{\circ} \mathrm{C}$. Following washing, the cells were resuspended in Annexin V staining buffer and $10-\mu \mathrm{g} / \mathrm{ml}$ PI $(10 \mu \mathrm{l})$ was added to each sample. Flow cytometry was immediately performed to measure U2-OS cell apoptosis using a FACSCalibur (BD Biosciences, Franklin Lakes, NJ, USA).

Terminal deoxynucleotidyl transferase dUTP nick end labeling (TUNEL) assay. A TUNEL assay was performed with a Fluorescein-FragEL ${ }^{\mathrm{TM}}$ DNA Fragmentation Detection kit (EMD Millipore, Gibbstown, NJ, USA) according to the manufacturer's instructions. Briefly, U2-OS cells were grown on glass slides and then fixed with $4 \%$ formaldehyde at $4{ }^{\circ} \mathrm{C}$ for $25 \mathrm{~min}$. This was followed by incubation with $0.2 \%$ Triton X-100 (Life Technologies, Grand Island, NY, USA) at room temperature for $5 \mathrm{~min}$. The slides were immersed in 1xTdT Equilibration buffer for $30 \mathrm{~min}$, and incubated with Fluorescein-TdT labeling mixture and TdT enzyme (Sigma-Aldrich) for $1 \mathrm{~h}$ at $37^{\circ} \mathrm{C}$. Staining was completed by a 1-min incubation with DAPI (Molecular Probes Life Technologies, Carlsbad, CA, USA) and the coverslips were mounted on slides. Measurements of TUNEL-positive nuclei were performed on 10-15 images/slide (captured by an independent observer blinded to the experiment) using a microscope (BX43; Olympus Corporation, Tokyo, Japan) connected to a digital camera, which was attached to an image processor (Quantity One 4.6.2; Bio-Rad Laboratories, Inc., Hercules, CA, USA). The images were recorded and saved using Adobe Photoshop 6 (Adobe Systems, San Jose, CA, USA).

Measurement of caspase-3 activity. Caspase-3 activity was assayed using a caspase-3 activity assay kit according to the manufacture's instructions. The assay is based on spectrophotometric detection of the chromophore p-nitroaniline (pNA) following cleavage from the labeled substrate, Ac-DEVD-pNA. The free pNA is quantified using a spectrophotometer or a microtiter plate reader at $405 \mathrm{~nm}$. Comparison of the absorbance of pNA from an apoptotic sample with an uninduced control allows determination of the fold increase in caspase- 3 activity. Briefly, U2-OS cells were digested, harvested and lysed with lysis buffer. Total proteins were measured using the BCA protein assay kit and the cell lysates were incubated with $0.2 \mathrm{mM}$ Ac-DEVD-pNA for $60-120 \mathrm{~min}$ at $37^{\circ} \mathrm{C}$. The OD values at $\mathrm{A} 405$ were measured by spectrophotometry detection (MK3; Tocan Trading Co., Ltd., Shanghai, China).

Western blotting. Total proteins from U2-OS cells were extracted using a lysis buffer containing $60 \mu \mathrm{g} / \mathrm{ml}$ phenylmethylsulfonyl fluoride (Tokyo Chemical Industry Co., Ltd., Tokyo, Japan). Protein concentrations were assessed using the BCA protein assay kit. The protein samples were denatured at $100^{\circ} \mathrm{C}$ for $5 \mathrm{~min}$ and separated by $10 \%$ SDS-PAGE (Life Technologies). After transferring and blocking with blocking buffer (1X Tris-buffered saline-Tween with 5\% w/v nonfat dry milk) for 1-2 $\mathrm{h}$, the membranes were hybridized with primary antibodies on a shaker (cat. no. 4637-1CECN; Thermo Fisher Scientific, Inc., Waltham, MA, USA) overnight at $4^{\circ} \mathrm{C}$. After washing, the membranes were incubated with a secondary antibodies on a shaker for $1 \mathrm{~h}$ at $37^{\circ} \mathrm{C}$. Enhanced chemiluminescence regents were used for detection. The films were developed using autographed films and scanned with a laser densiDetect (Applied Biosystems Life Technologies).

Statistical analysis. Statistical analysis was performed using Student's t-test. $\mathrm{P}<0.05$ was considered to indicate a statistically significant difference and P-values were two-tailed.

\section{Results}

PEITC and ADM treatment inhibits proliferation of U2-OS cells in a dose-dependent manner. To investigate the synergistic treatment effect of PEITC and ADM on osteosarcoma cells, the $\mathrm{IC}_{50}$ value of PEITC or ADM against U2-OS cells was assessed. U2-OS cells were treated with a series of doses of PEITC or ADM for $24 \mathrm{~h}$ and the OD values were measured using an MTT assay. As shown in Fig. 1A, PEITC 
Table I. Effects of ADM and PEITC on osteosarcoma cells.

\begin{tabular}{|c|c|c|c|c|c|c|c|c|c|}
\hline Group & Conc. ${ }^{a}$ & Blank & OD1 & OD2 & OD3 & Mean & $\begin{array}{l}\text { Adj. OD } \\
\text { value }\end{array}$ & $\begin{array}{c}\text { Inhibition } \\
\text { rate }(\%)\end{array}$ & Q-value \\
\hline Untreated & 0 & 0.097 & 0.376 & 0.399 & 0.407 & 0.394 & 0.297 & - & - \\
\hline ADM & 1 & 0.091 & 0.344 & 0.329 & 0.324 & 0.332 & 0.241 & 18.743 & - \\
\hline PEITC & 2 & 0.119 & 0.346 & 0.356 & 0.327 & 0.343 & 0.224 & 24.579 & - \\
\hline ADM + PEITC & $1+2$ & 0.121 & 0.255 & 0.281 & 0.254 & 0.263 & 0.142 & 52.076 & 1.345 \\
\hline ADM & 3 & 0.102 & 0.333 & 0.323 & 0.324 & 0.327 & 0.225 & 24.242 & - \\
\hline PEITC & 2.5 & 0.105 & 0.330 & 0.327 & 0.312 & 0.323 & 0.218 & 26.599 & - \\
\hline ADM + PEITC & $3+2.5$ & 0.118 & 0.204 & 0.245 & 0.214 & 0.221 & 0.103 & 65.320 & 1.471 \\
\hline ADM & 10 & 0.134 & 0.308 & 0.297 & 0.303 & 0.303 & 0.169 & 43.210 & - \\
\hline PEITC & 4 & 0.099 & 0.277 & 0.253 & 0.255 & 0.262 & 0.163 & 45.230 & - \\
\hline ADM + PEITC & $10+4$ & 0.098 & 0.179 & 0.193 & 0.181 & 0.184 & 0.086 & 70.887 & 1.029 \\
\hline
\end{tabular}

${ }^{a}$ Units: ADM, $\mu \mathrm{g} / \mathrm{ml}$; PEITC, $\mu \mathrm{M}$. ADM, adriamycin; PEITC, phenethyl isothiocyanate; Conc., concentration; OD, optical density.

A

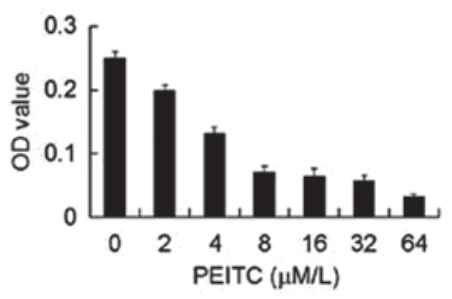

C

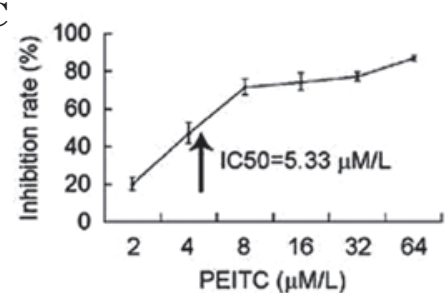

B

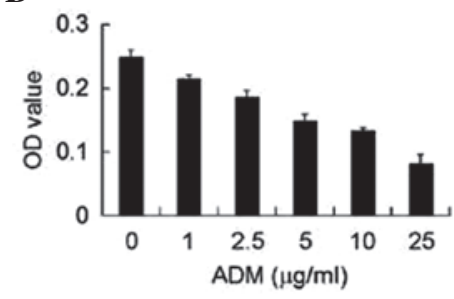

D

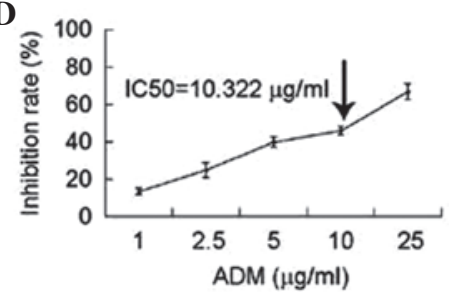

Figure 1. Effect of ADM and PEITC on proliferation of U2-OS cells. Graphs showing OD values of U2-OS cells treated with a series of (A) PEITC or (B) ADM doses as measured by MTT assay $(n=3)$. Graphs showing inhibition rate of (C) PEITC or (D) ADM on U2-OS cells. The inhibition rate was calculated as follows: (OD value - OD baseline)/OD value x 100 . OD, optical density; PEITC, phenethyl isothiocyanate; ADM, adriamycin; $\mathrm{IC}_{50}$, half maximal inhibitory concentration.

and ADM had a dose-dependent effect on U2-OS cells. The OD values decreased as the dose of PEITC or ADM increased. When the OD values were converted into inhibition rates [(OD value - OD baseline)/OD value x 100], PEITC and ADM were found to dose-dependently inhibit the proliferation of U2-OS cells. SPSS Statistics 17.0 software (SPSS, Inc., Chicago, IL, USA) was used to determine that the concentration of PEITC and ADM, which caused 50\% inhibition of U2-OS cells (the $\mathrm{IC}_{50}$ value) was $5.33 \mu \mathrm{M}$ and $10.322 \mu \mathrm{g} / \mathrm{ml}$, respectively.

Following this, whether PEITC and ADM treatment exerts synergistic, additive or antagonistic effects on U2-OS cells was determined. The cells were treated with PEITC and ADM alone or with a combination of the two at varying concentrations for $24 \mathrm{~h}$, and the OD values were measured by MTT assay. The untreated cells served as a control when

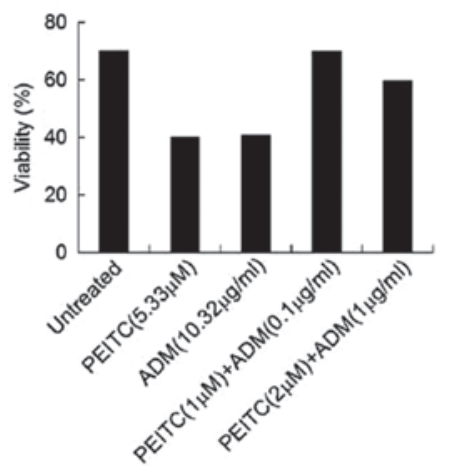

Figure 2. Effect of ADM and PEITC on viability of U2-OS cells. The viability of U2-OS cells treated with either PEITC or ADM or a combination of the two. The viability was measured by Annexin V and PI staining, and analyzed by flow cytometry. The Annexin V/PI-stained cells were the viable cells and these were counted to calculate the percentage of viability. PEITC, phenethyl isothiocyanate; ADM, adriamycin; PI. propidium iodide. 
A

DAPI
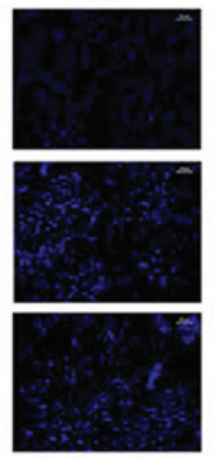

B DAPI
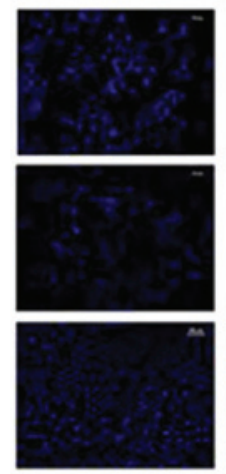

TUNEL
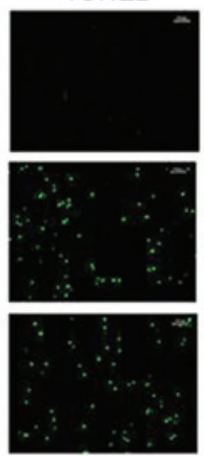

TUNEL
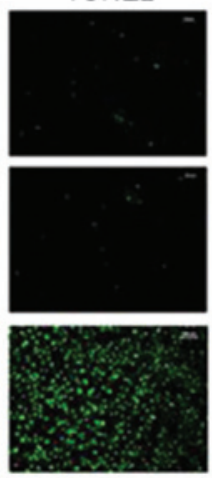

Merge
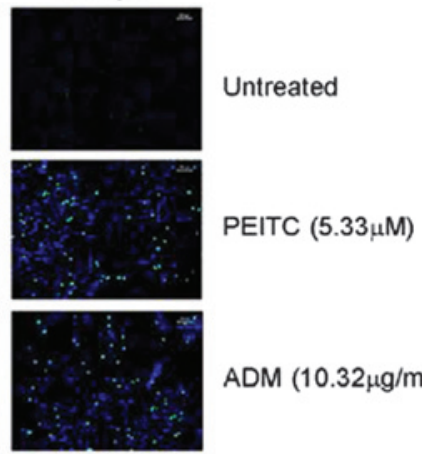

$\operatorname{ADM}(10.32 \mu \mathrm{g} / \mathrm{ml})$
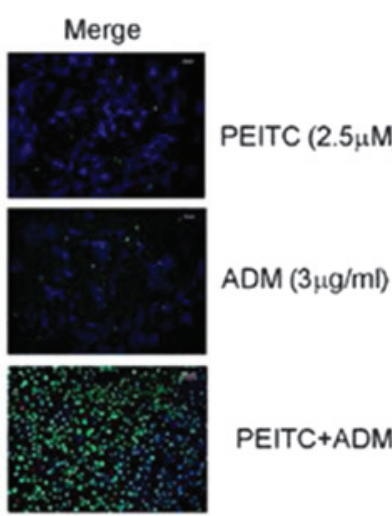

$\operatorname{ADM}(3 \mu \mathrm{g} / \mathrm{ml})$
PEITC+ADM

C

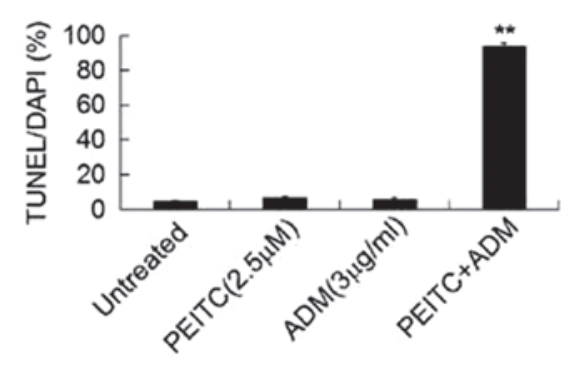

Figure 3. Effect of ADM and PEITC on apoptosis of U2-OS cells. (A) Representative images showing apoptosis of U2-OS cells by TUNEL assay. U2-OS cells were treated with the half maximal inhibitory concentration of PEITC or ADM. DAPI staining was used to identify cells; the fluorescence-stained cells were apoptotic. (B) Representative images showing apoptosis of U2-OS cells treated with $2.5 \mu \mathrm{M}$ PEITC or $3 \mu \mathrm{g} / \mathrm{ml}$ ADM alone, or in combination. (C) Summary of the percentage of TUNEL cells from DAPI-stained cells out of the U2-OS cells that were treated with either $2.5 \mu \mathrm{M}$ PEITC or $3 \mu \mathrm{g} / \mathrm{ml}$ ADM or a combination of the two $(\mathrm{n}=4)$. ${ }^{* *} \mathrm{P}<0.01$, compared with either untreated cells, or cells treated wi th PEITC or ADM alone. PEITC, phenethyl isothiocyanate; ADM, adriamycin; TUNEL, terminal deoxynucleotidyl transferase dUTP nick end labeling.

calculating the inhibition rates of PEITC or ADM. As shown in Table I, while treatment with each therapeutic agent alone dose-dependently produced a substantial inhibition rate of the U2-OS cells, a combination of the two therapeutic agents led to an increased inhibition rate when compared with either PEITC or ADM treatment alone. Q-values were calculated using the following formula: $\mathrm{Q}=\mathrm{Ea}+\mathrm{b} /(\mathrm{Ea}+\mathrm{Eb}-\mathrm{Ea} \times \mathrm{Eb}) . \mathrm{Ea}$ and $\mathrm{Eb}$ represent the inhibition rate of $\mathrm{ADM}$ and PEITC alone, respectively; $\mathrm{Ea}+\mathrm{b}$ represents the inhibition rate of $\mathrm{ADM}$ and PEITC in combination. A Q-value $>1.15$ signifies a synergistic effect; a Q-value $>0.85$ and $<1.15$ signifies an additive effect
A

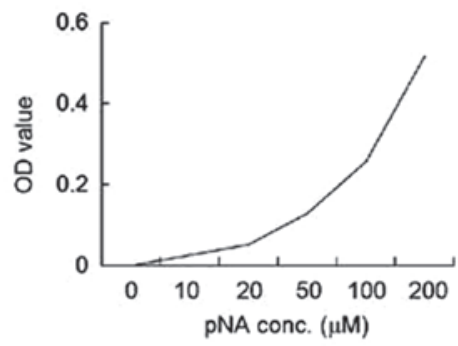

B

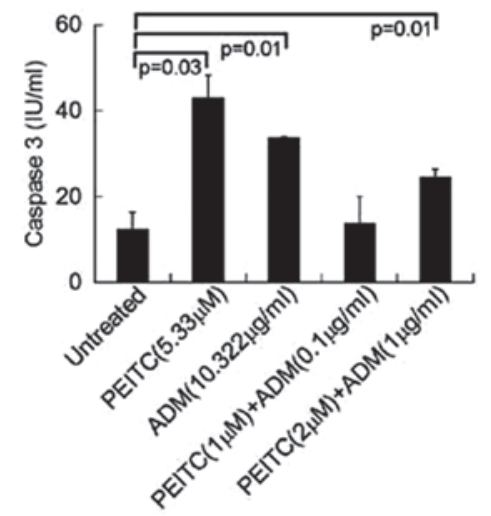

Figure 4. Effect of PEITC and ADM on caspase-3 activity of U2-OS cells (A) Standard curve presenting the OD values of increasing doses of pNA detected by spectrophotometry. (B) Caspase-3 activity of lysates of U2-OS cells treated with either PEITC or ADM, or a combination of the two. The OD values at $\mathrm{A} 405$ were measured by spectrophotometry and used to calculate the activity of caspase-3 $(n=3)$. OD, optical density; pNA, chromophore p-nitroaniline; PEITC, phenethyl isothiocyanate; ADM, adriamycin.

and a $\mathrm{Q}$-value $<0.85$ signifies an antagonistic effect. Based on this, the combination of low doses of PEITC and ADM was observed to produce a synergistic effect, while the combination of high doses of the two therapeutic agents resulted in an additive effect (Table I). No antagonistic effect was observed for PEITC + ADM treatment.

Treatment with PEITC and ADM alone and in combination increases U2-OS cell apoptosis. Given the inhibitory effects of PEITC or ADM observed in the osteosarcoma cells, whether PEITC and ADM alone or in combination impacted survival of osteosarcoma cells was subsequently investigated. To do this, U2-OS cells were stained with Annexin V and PI and the U2-OS cell apoptosis was measured. The viable cells were defined as Annexin $\mathrm{V}^{-}$and $\mathrm{PI}^{-}$cells. As shown in Fig. 2, $\mathrm{U} 2-\mathrm{OS}$ cells treated with the $\mathrm{IC}_{50}$ concentration of PEITC or ADM displayed a decreased number of viable cells [viability from $70 \%$ (observed in the untreated cells) to 40\%]. The findings demonstrate that a combination treatment of PEITC and ADM dose-dependently marginally decreased the viability of U2-OS cells.

In addition to Annexin V/PI staining, a TUNEL assay was conducted to determine the apoptotic DNA fragmentation. A fluorescence-labeled TdT was incubated with fixed and permeabilized U2-OS cells and the apoptotic cells were visualized using a fluorescent microscope (cells exhibiting fluorescence were apoptotic). Analysis of the TUNEL assay of the U2-OS cells treated with or without the $\mathrm{IC}_{50}$ concentration of $\mathrm{ADM}$ 


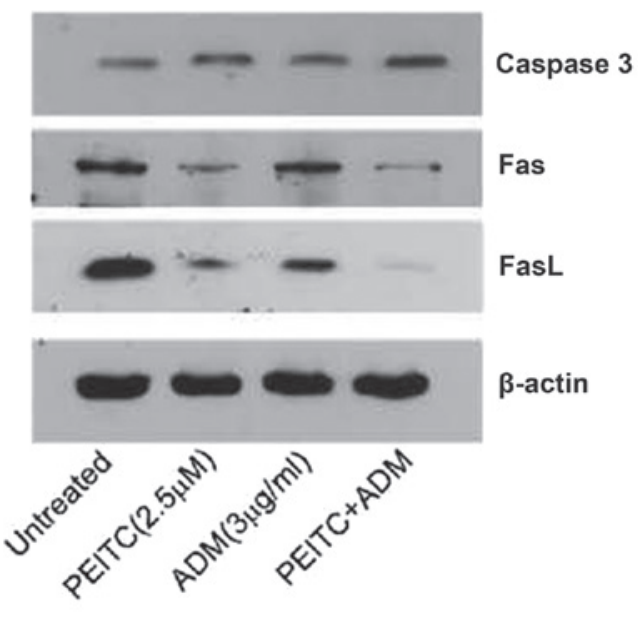

Figure 5. Effect of PEITC and ADM on the apoptotic signaling pathway of U2-OS cells. Representative gel images showing expression of caspase-3, Fas, FasL or $\beta$-actin in U2-OS cells treated with either PEITC or ADM, or a combination of the two. Expression of caspase-3, Fas or FasL was measured by western blotting $(n=2)$. PEITC, phenethyl isothiocyanate; ADM, adriamycin; FasL, Fas ligand.

or PEITC was conducted first. As shown in Fig. 3A, while the viability of the untreated U2-OS cells was high, treatment with $5.33 \mu \mathrm{M}$ PEITC or $10.322 \mu \mathrm{g} / \mathrm{ml}$ ADM significantly induced U2-OS cell apoptosis, as indicated by the increased number of fluorescent cells. Subsequently, the effect of the combination treatment (ADM + PEITC) at lower doses on TUNEL in the U2-OS cells was assessed. Treatment with $2.5 \mu \mathrm{M}$ PEITC or $3 \mu \mathrm{g} / \mathrm{ml}$ ADM marginally increased TUNEL in the U2-OS cells; however, a combination of the two therapeutic agents markedly enhanced the number of TUNEL cells (Fig. 3B). The percentage of TUNEL cells from DAPI-stained cells was then quantified and calculated. The data from three experiments is summarized in Fig. 3C. As expected, treatment with a combination of PEITC and ADM significantly increased the percentage of TUNEL cells when compared with the untreated group or with the cells treated with PEITC or ADM alone (Fig. 3C).

Treatment with PEITC and ADM alone and in combination increases caspase-3 activity in U2-OS cells. Caspase-3 is key in mediating cell apoptosis and the increased activity of caspase- 3 causes or is the result of apoptosis. To further determine the apoptotic induction effect of PEITC and ADM on U2-OS cells, U2-OS cells were treated with PEITC and ADM to determine whether they activate caspase-3 and increase its activity. A caspase-3 activity assay kit was used and a linear standard curve was produced, which presents the increased OD values against increasing concentrations of pNA (Fig. 4A). Subsequently, caspase-3 activity of the U2-OS cells treated with the $\mathrm{IC}_{50}$ concentration of PEITC or ADM was measured (untreated cells served as the control). As shown in Fig. 4B, treatment with PEITC and ADM alone at the $\mathrm{IC}_{50}$ concentration significantly increased capspase-3 activity in U2-OS cells compared with the untreated cells $(\mathrm{P}<0.05)$. The effect of the combination treatment $(\mathrm{PEITC}+\mathrm{ADM})$ at decreased doses on caspase-3 activity in U2-OS cells was assessed. Although a combination of PEITC $(1 \mu \mathrm{M})$ and $\mathrm{ADM}(0.1 \mu \mathrm{g} / \mathrm{ml})$ marginally increased caspase-3 activity, $2 \mu \mathrm{M}$ PEITC combined with $1 \mu \mathrm{g} / \mathrm{ml}$ ADM significantly enhanced caspase- 3 activity $(\mathrm{P}<0.05)$.

Effect of PEITC and ADM treatment on the apoptotic signaling pathway of U2-OS cells. As PEITC and ADM treatment significantly increased caspase-3 activity, whether caspase-3 expression was induced in U2-OS cells treated with PEITC and ADM was evaluated. Western blot analysis revealed that while treatment with $2.5 \mu \mathrm{M}$ PEITC or $3 \mu \mathrm{g} / \mathrm{ml}$ ADM alone increased the expression of caspase- 3 protein, a combination treatment with the two therapeutic agents further upregulated caspase-3 expression in the U2-OS cells. In addition, the expression of Fas and Fas ligand (FasL) was observed to decrease in U2-OS cells treated with either PEITC and ADM alone or in combination, indicating that Fas/FasL may not be involved in PEITC/ADM-induced apoptosis.

\section{Discussion}

The significant finding of the present study is that ADM achieves a comparable effect in osteosarcoma cells at a lower dose when incubated with PEITC. This finding is particularly important as drug resistance and side-effects due to the administration of high doses of ADM lead to failure of this cancer therapy.

In the present study, PEITC synergistically enhanced the ADM-mediated inhibition of U2-OS cell proliferation. The $\mathrm{IC}_{50}$ values of PEITC and ADM against the U2-OS cells were identified to be $5.33 \mu \mathrm{M}$ and $10.322 \mu \mathrm{g} / \mathrm{ml}$, respectively. However, when incubated together, the $\mathrm{IC}_{50}$ values of PEITC and ADM against U2-OS cells significantly decreased to a dose $<2 \mu \mathrm{M}$ and $1 \mu \mathrm{g} / \mathrm{ml}$, respectively (data not shown). Clinical administration of a reduced dose of ADM, which achieves a comparable effect to its original dose, is considered to be beneficial to patient outcome.

As a chemotherapeutic agent, ADM induces apoptosis in various types of cancer cell. Similar to ADM, PEITC treatment has been shown to induce apoptosis in tumor cells (19). Supporting these findings, the in vitro results of the present study demonstrate that cell viability was low in U2-OS cells that were treated with either ADM or PEITC. When administered in combination, the rate of apoptosis in U2-OS cells significantly increased when compared with cells treated with ADM or PEITC alone. These results indicate that PEITC sensitizes U2-OS cells to ADM-induced apoptosis, which is consistent with previous studies $(20,21)$.

Various signaling pathways have been proposed to be involved in ADM-induced apoptosis of cancer cells. For example, it has been shown that ADM induces Fas-mediated apoptosis in human thyroid carcinoma cells (22); however, a study found that ADM-induced apoptosis was primarily dependent on tumor necrosis factor-related apoptosis-inducing ligand (TRAIL)/TRAIL-receptor signaling, although not on FasL, perforin, NKG2D or DNAX Accessory Molecule-1 (23). No increased expression of Fas/FasL was noted in U2-OS cells that were treated with PEITC and ADM, indicating that the Fas/FasL signaling pathway is not involved in ADM-induced apoptosis in U2-OS cells.

Although Fas/FasL was not found to be involved in U2-OS cells treated with PEITC and ADM, the activity and expression of caspase-3 were found to be significantly enhanced 
in PEITC- and ADM-treated U2-OS cells. While this result validates the finding that PEITC and ADM treatment induces apoptosis of U2-OS cells due to upregulation of caspases, including caspase-3, cause or is the result of apoptosis (24), it remains unknown which apoptotic signaling pathway is involved in PEITC/ADM-induced apoptosis in U2-OS cells. Therefore, further investigation is required.

In conclusion, PEITC and ADM treatment synergistically inhibited the proliferation of U2-OS cells. In addition to proliferation inhibition, PEITC and ADM treatment synergistically induced the apoptosis of U2-OS cells. This induction was associated with activity elevation and upregulated expression of caspase-3. These findings determined the biological effects of PEITC and ADM treatment on U2-OS cells, as well as revealing the therapeutic potential of treatment with PEITC in conjunction with ADM in patients exhibiting osteosarcoma.

\section{References}

1. Dotan A, Dadia S, Bickels J, Nirkin A, Flusser G, Issakov J, Neumann Y, Cohen I, Ben-Arush M, Kollender Y, et al: Expandable endoprosthesis for limb-sparing surgery in children: Long-term results. J Child Orthop 4: 391-400, 2010.

2. Broadhead ML, Clark JC, Myers DE, Dass CR and Choong PF: The molecular pathogenesis of osteosarcoma: A review. Sarcoma 2011: 959248, 2011

3. Ottaviani G and Jaffe N: The epidemiology of osteosarcoma. Cancer Treat Res 152: 3-13, 2009.

4. Ottaviani $\mathrm{G}$ and Jaffe N: The etiology of osteosarcoma. Cancer Treat Res 152: 15-32, 2009

5. Weiss RB: The anthracylcines: Will we ever find a better doxorubicin? Semin Oncol 19: 670-686, 1992.

6. Thorna CF, Oshiroa C, Marshe S, Hernandez-Boussard T, McLeod H, Kleina TE and Altman RB: Doxorubicin pathways: Pharmacodynamics and adverse effects. Pharmacogenet Genomics 21: 440-446, 2011.

7. Beklemisheva AA, Fang Y, Feng J, Ma X, Dai W and Chiao JW: Epigenetic mechanism of growth inhibition induced by phenylhexyl isothiocyanate in prostate cancer cells. Anticancer Res 26: 1225-1230, 2006.

8. Ma X, Fang Y, Beklemisheva A, Dai W, Feng J, Ahmed T, Liu D and Chiao JW: Phenylhexyl isothiocyanate inhibits histone deacetylases and remodels chromatins to induce growth arrest in human leukemia cells. Int J Oncol 28: 1287-1293, 2006.

9. Lu Q, Lin X, Feng J, Zhao X, Gallagher R, Lee MY, Chiao JW and Liu D: Phenylhexyl isothiocyanate has dual function as histone deacetylase inhibitor and hypomethylating agent and can inhibit myeloma cell growth by targeting critical pathways. J Hematol Oncol 9: 1-6, 2008.
10. Stan SD, Singh SV, Whitcomb DC and Brand RE: Phenethyl isothiocyanate inhibits proliferation and induces apoptosis in pancreatic cancer cells in vitro and in a MIAPaca2 xenograft animal model. Nutr Cancer 66: 747-755, 2014, Nov 6 2013. [Epub ahead of print].

11. Huang SH, Hsu MH, Hsu SC, Yang JS, Huang WW, Huang AC, Hsiao YP, Yu CC and Chung JG: Phenethyl isothiocyanate triggers apoptosis in human malignant melanoma A375.S2 cells through reactive oxygen species and the mitochondria-dependent pathways. Hum Exp Toxicol 33: 270-283, 2014.

12. Sarkars R, Mukherjee S and Roy M: Targeting heat shock proteins by phenethyl isothiocyanate results in cell-cycle arrest and apoptosis of human breast cancer cells. Nutr Cancer 65: 480-493, 2013.

13. Xiao D and Singh SV: p66Shc is indispensable for phenethyl isothiocyanate-induced apoptosis in human prostate cancer cells. Cancer Res 70: 3150-3158, 2010.

14. Cang S, Ma Y, Chiao JW and Liu D: Phenethyl isothiocyanate and paclitaxel synergistically enhanced apoptosis and alpha-tubulin hyperacetylation in breast cancer cells. Exp Hematol Oncol 3: 5, 2014.

15. Lee DH, Kim DW, Lee HC, Lee JH and Lee TH: Phenethyl isothiocyanate sensitizes glioma cells to TRAIL-induced apoptosis. Biochem Biophys Res Commun 446: 815-521, 2014.

16. Tang T, Song X, Liu YF and Wang WY: PEITC reverse multi-drug resistance of human gastric cancer SGC7901/DDP cell line. Cell Biol Int 38: 502-510, 2014.

17. Liu K, Cang S, Ma Y and Chiao JW: Synergistic effect of paclitaxel and epigenetic agent phenethyl isothiocyanate on growth inhibition, cell cycle arrest and apoptosis in breast cancer cells. Cancer Cell Int 13: 10, 2013.

18. Xiao D and Singh SV: Phenethyl isothiocyanate sensitizes androgen-independent human prostate cancer cells to docetaxel-induced apoptosis in vitro and in vivo. Pharm Res 27: 722-731, 2010.

19. Gupta P and Srivastava SK: Antitumor activity of phenethyl isothiocyanate in HER2-positive breast cancer models. BMC Med 10: 80, 2012.

20. Yuang P, Chen BA, Cheng J, Ma XD, Liu DL and Lu QY: Effect of phenylhexyl isothiocyanate on adriamycin resistance of K562/A02 cell line. Zhongguo Shi Yan Xue Ye Xue Za Zhi 17: 352-357, 2009 (In Chinese).

21. Mukherjee S, Bhattacharya RK and Roy M: Targeting protein kinase $\mathrm{C}$ (PKC) and telomerase by phenethyl isothiocyanate (PEITC) sensitizes PC-3 cells towards chemotherapeutic drug-induced apoptosis. J Environ Pathol Toxicol Oncol 28: 269-282, 2009.

22. Massart C, Barbet R, Genetet N and Gibassier J: Doxorubicin induces Fas-mediated apoptosis in human thyroid carcinoma cells. Thyroid 14: 263-270, 2004.

23. Wennerberg E, Sarhan D, Carlsten M, Kaminskyy VO, D'Arcy P, Zhivotovsky B, Childs R and Lundqvist A: Doxorubicin sensitizes human tumor cells to NK cell- and T-cell-mediated killing by augmented TRAIL receptor signaling. Int J Cancer 133: 1643-1652, 2013.

24. Degterev A, Boyce M and Yuan J: A decade of caspases. Oncogene 22: 8543-8567, 2003. 